\title{
Making a Silk Purse Out of Two Sow's Ears: Young Children's Use of Comparison in Category Learning
}

\author{
Laura L. Namy \\ Emory University
}

\author{
Dedre Gentner \\ Northwestern University
}

\begin{abstract}
Comparison mechanisms have been implicated in the development of abstract, relational thought, including object categorization. D. Gentner and L. L. Namy (1999) found that comparing 2 perceptually similar category members yielded taxonomic categorization, whereas viewing a single member of the target category elicited shallower perceptual responding. The present experiments tested 2 predictions that follow from Gentner and Namy's (1999) model: (a) Comparison facilitates categorization only when the targets to be compared share relational commonalities, and (b) providing common labels for targets invites comparison, whereas providing conflicting labels deters it. Four-year-olds participated in a forced-choice task. They viewed 2 perceptually similar target objects and were asked to "find another one." Results suggest an important role for comparison in lexical and conceptual development.
\end{abstract}

Children are phenomenally effective word learners. They gain new words at an astonishing rate. Research aimed at explicating the processes by which children accomplish this feat of learning has engendered a paradoxical set of findings. On the one hand, there is considerable evidence that children's categorization of objects is conceptually driven. Young children often categorize on the basis of deep, nonobvious conceptual aspects of objects such as function (e.g., can be eaten), casual properties (e.g., has eyes so it can see), and relations to other things in the world (e.g., grows on trees) (Golinkoff, Hirsh-Pasek, Bailey, \& Wenger, 1992; Kemler Nelson, 1995; E. M. Markman, 1989; E. M. Markman \& Hutchinson, 1984; Waxman \& R. Gelman, 1986; Waxman \& Kosowski, 1990), and can also make inferences on the basis of such properties (Gelman \& Coley, 1990; S. A. Gelman \& E. M. Markman, 1986; S. A. Gelman \& Wellman, 1991; Kalish \& S. A. Gelman, 1992). In striking contrast to studies demonstrating children's appreciation of the nonobvious aspects of category organization, many studies of children's early naming suggest that children often rely primarily on perceptual features such as shape (e.g., round) and distinctive features (e.g., wheels) rather than on conceptual knowl-

Laura L. Namy, Department of Psychology, Emory University; Dedre Gentner, Department of Psychology, Northwestern University.

This project was supported by an Emory University Research Grant to Laura L. Namy and by National Science Foundation Grant SBR-9511757 to Dedre Gentner. We are also grateful for the financial support provided by William T. Grant Foundation Award 95167795 . This article was partially prepared while Dedre Gentner was a Fellow at the Center for Advanced Study in the Behavioral Sciences. We thank Marjorie Jones and Michelle Osmondson for assistance with stimulus design and data collection. We also thank Jeff Loewenstein and the entire Similarity and Analogy group at Northwestern University for discussions of this research. Finally, we are grateful to the children and parents who participated in the experiments.

Correspondence concerning this article should be addressed to Laura $\mathrm{L}$. Namy, Department of Psychology, Emory University, Atlanta, Georgia 30322. E-mail: Lnamy@emory.edu edge as a basis for categorization (Baldwin, 1992; Bowerman, 1976; Clark, 1973; Cohen \& Oakes, 1993; Gentner, 1978; Gentner \& Imai, 1995; Golinkoff et al., 1992; Imai, Gentner, \& Uchida, 1994; Landau, Smith, \& Jones, 1988, 1998; Rakison \& Butterworth, 1998; Smith, Jones, \& Landau, 1992; Tomikawa \& Dodd, 1980; Ward et al., 1989). Clark (1973) was among the first to document that children frequently made shape-based extensions in their early spontaneous word use, as recorded in parental diaries. She noted that children often extended words to objects that were conceptually unrelated and appeared in very different contexts but had the same shape. Bowerman (1976) also observed perceptually based overgeneralizations in children's early language, such as using the word moon to describe any crescent-shaped object ranging from a moon to a half grapefruit to a hangnail. Similarly, Smith, Landau, and Jones (Landau et al., 1988, 1998; Smith et al., 1992) have shown that young children often extend novel words by shape.

Gentner's (1978) "Jiggy/Zimbo" study provided the first experimental examination of the relative contribution of perceptual appearance and conceptual knowledge to word extension. She demonstrated that children extended novel words to objects on the basis of perceptual appearance rather than function even when the functional properties of the object were extremely salient (e.g., it dispenses candy; see also Landau et al., 1998, but cf. with Kemler Nelson, 1995, 1999, discussed later).

More recently, Baldwin $(1989,1992)$ specifically examined the issue of perceptual versus taxonomic responding. She taught preschool-aged children a new name for a pictured object (e.g., an egg) and then asked them to choose another object that would receive the same name. She found that children were equally likely to select either a perceptually dissimilar member of the same taxonomic category (e.g., a loaf of bread) or a perceptually similar member of a different category (e.g., a football) despite the fact that these object categories were familiar to the children. In fact, children who were given a choice between a perceptually similar object that was entirely unrelated to the target (e.g., a football) and 
a thematically related object (e.g., a nest) overwhelmingly selected the unrelated object as a match for the target on the basis of its perceptual similarity.

In a similarly structured task, Imai et al. (1994) found that 3-year-olds and 5-year-olds who were taught a novel name for an object (e.g., an apple) tended to extend the word to a perceptually similar object from a different object category (e.g., a balloon) over another perceptually distinct member that was of the same category as the standard (e.g., a banana). In fact, they found that even when children were given a choice between an object that shared both appearance and category membership with the target (e.g., a pear) and an object that shared only appearance (e.g., a balloon), children were equally likely to select either object. Clearly, then, a large number of studies have converged to demonstrate that perceptual properties such as shape loom large in children's responses on categorization tasks. This evidence suggests that children rely on shape or other salient perceptual features-perhaps even to an extent that seems detrimental to their acquisition of conceptually coherent object categories.

How can this clear evidence that children are perceptually oriented in their categorization behaviors be reconciled with the compelling findings that children form rich conceptual categories? To address this issue, we considered more carefully the processes of forming and using categories. Specifically, we have proposed that comparison, the act of examining two or more like things in conjunction to assess commonalities and differences, can provide a resolution to this empirical conflict. We proposed first that hearing common labels applied to multiple entities invites children to engage in comparison processes, and second, that the process of comparison highlights additional conceptual commonalities that are not immediately evident on surface-level inspection.

In what follows, we review evidence demonstrating the benefit of comparison in adults' and children's learning. In the following section, we consider the question of whether structural alignment mechanisms in particular-as opposed to a more traditional similarity-based account-best explain these learning phenomena. We describe how our prior research addresses this question and supports the claim that comparison provides a resolution to the conflicting empirical findings regarding the basis of children's categorization.

\section{The Effects of Comparison on Learning}

The proposal that comparison can promote deep learning is based on Gentner's structure-mapping theory of comparison (1983, 1989; Falkenhainer, Forbus, \& Gentner, 1989; Gentner \& Markman, 1997; Wolff \& Gentner, 2000). According to structuremapping theory, comparison acts to highlight commonalities that may not have been explicitly accessed prior to comparison. In particular, the process of aligning two representations can result in the extraction of common higher order relational structure that was not readily evident within either item alone.

Positive effects of comparison on learning are well established in studies of adult learning (Gentner \& Markman, 1995, 1997; Gentner \& Wolff, 1997; Gick \& Holyoak, 1983; Holyoak \& Thagard, 1989; Hummel \& Holyoak, 1997; Lassaline \& Murphy, 1998; A. B. Markman, 1997; Medin, Goldstone, \& Gentner, 1993; Ross \& Spalding, 1991; Wolff \& Gentner, 2000). Gick and Ho- lyoak (1983) provided one of the earliest demonstrations of the facilitative effects of comparison on learning in adults. They gave college students a difficult problem to solve. Prior to solving the problem, the students either read one analogically similar story or were instructed to compare two analogically similar stories. Students who compared the two stories and wrote out the commonalities between them were far more likely to solve the later problem than those who simply read one story. Similarly, Loewenstein, Thompson, and Gentner (1999) documented that business school students who engaged in explicit comparison of two negotiation scenarios were more apt to apply appropriate negotiation strategies in real world situations than were those who studied the same two scenarios without explicitly comparing them. A. B. Markman and Gentner (1993) also found that eliciting comparison enabled adults to focus on abstract relational commonalities among scenes. It is important to note that the higher order commonalities discovered by the participants in these studies were not plucked out of the air. Rather, the comparison process takes as its starting point the literal or surface-level similarities and uses these similarities as a springboard into related abstract or relational commonalities derived from the perceptual commonalities. Gentner et al. (1997) refer to this alignment process as the "comparison-as$\mathrm{X}$-ray" phenomenon.

Recent literature on conceptual development suggests that this comparison mechanism is at work in children's learning processes as well. Children who are encouraged to engage in comparison often succeed at more difficult or more abstract tasks than children who have not engaged in comparison. This effect of comparison in promoting abstract knowledge is evident across a wide span of ages and cognitive tasks. For example, Loewenstein and Gentner (2001) found a comparison benefit on preschool children's ability to perform a spatial-mapping task from one model room to another. Asking children to explicitly compare and identify correspondences between two model rooms facilitated transfer of spatial correspondence to a third room. Waxman and Klibanoff (2000; Klibanoff \& Waxman, 2000; Waxman, 1999) have found that comparing like objects facilitates infants' and preschoolers' ability to map adjectives to the correct referent object property (such as color or texture). Gentner and colleagues (Gentner, Rattermann, Markman, \& Kotovsky, 1995; Kotovsky \& Gentner, 1996) have also demonstrated that comparison heightens children's ability to ignore compelling perceptual commonalities in favor of relational commonalities. Finally, R. Gelman (1969) demonstrated that inducing children to compare between domains in which they understand conservation and those in which they do not yet grasp the principle can lead to gains in understanding conservation in the latter domains.

Even in studies with infants, researchers have observed that comparison can benefit object recognition and discrimination. For example, infants perform better on a facial recognition task if they are given the opportunity to compare the target face from two different angles (Fagan, 1978). More relevant to the acquisition of categories, a study by Oakes (2001) recently demonstrated that 4 to 6-month-old infants more readily form perceptual categories such as "dog" (and discriminate dogs from perceptually similar cats) when the infants are given the opportunity to view and compare objects in pairs than when the objects are presented one at a time. 


\section{How Structure Mapping Facilitates Children's Categorization}

We propose that the structural alignment model specified above may be particularly well suited to facilitating category formation in young children by inviting attention to deep, relational properties rather than surface-level, perceptual properties. Because infants and young children have relatively sparse and inexplicit knowledge of conceptual features of objects, they are easily captured by salient perceptual properties such as shape. However, comparison among exemplars can act to highlight relational commonalities (Gentner \& Medina, 1998; Gentner \& Namy, 1999; Gentner \& Rattermann, 1991). In this manner, we suggest, comparison fosters the development of more abstract conceptual knowledge of word meaning. Furthermore, we suggest that this effect of comparison on the mapping of words to categories is one manifestation of a domain-general learning process that manifests itself similarly in the additional domains described above.

Gentner and Namy (1999) provided evidence that structural alignment in particular, and not merely an overall similaritygeneralization process, operates to inform word learning in preschoolers. In that study, we sought to determine whether comparing instances of a category enables children to move beyond compelling perceptual commonalties to extract deeper conceptual object categories. As in the standard word extension task, children were taught a new name for an object (e.g., an apple) and then were asked to select which of two alternatives would have the same name as the standard: a perceptually distinct match from the same category (e.g., a banana) or a perceptually similar object from a different category (e.g., a balloon). Our innovation in these studies was to vary whether children saw a single standard (e.g., an apple) or multiple standards (e.g., an apple and a pear) from an object category. The stimuli were designed such that children tended to select the perceptual match when given either of the two standards by themselves. For example, when given either the apple or the pear, children chose the balloon rather than the banana. That is, either standard by itself led to the perceptual choice.

The key question is what happens when children are given both the apple and pear as standards and are invited to compare them. Note that the two standards both share the same (perceptual) features with the perceptual alternative. Thus, on traditional accounts of similarity, comparing the two standards should lead to an even greater reliance on the common (perceptual) features than was found for the single standards. Consequently, the preference for the perceptual match should be stronger in the comparison case than in the single-alternative case.

However, on a structure-mapping account, engaging in a comparison process can actually shift the basis for preference. This is because comparison leads to alignment of common relational structure as well as common surface features, thereby promoting the relations' salience. Because relations (even core relations such as internal causal or function relations) tend to be less accessible than object features, this alignment process will render relational commonalities more obvious in the pair than in either of the separate exemplars. In other words, the relations "stand more to gain" from this alignment process than do more obvious object properties.

If comparison renders relations more salient and if common labels encourage comparison, then this process might enable chil- dren to override compelling perceptual commonalities in favor of deeper conceptual ones. This is precisely what we found (Gentner \& Namy, 1999). When shown both standards together, children chose the conceptual match despite preferring the perceptual match for either of the standards presented singly. This outcome provides critical evidence that comparison facilitates categorization on the basis of conceptual, and not merely perceptual, features.

This remarkable finding that even when both standards individually support a perceptual choice the two together can be aligned to reveal a common conceptual basis of responding suggests a reconciliation of the perceptual-conceptual controversy discussed above. Seen in this light, children's use of perceptual features as a basis for categorization may in fact be a constructive heuristic when faced with little information about a category. Perceptual commonalities serve as the initial "hook" that encourages children to engage in comparison and extract deeper relational commonalities. Such relational commonalities may include common function (e.g., both are edible), mechanical causal relations (e.g., both are strong, so they can bend things) biological causal relations (e.g., both need water to grow), role relations (e.g., both grow on trees), and progeneration (e.g., both have babies that look like the adults but smaller). Clearly, apprehending these relationships is fundamental to the child's construction of a deep and coherent knowledge.

On the basis of this finding, Gentner and Namy (1999) argued that alignment among a word's referents on the basis of perceptual commonalities promotes attention to relational commonalities that can then serve to guide further word extensions. For example, the salience of the similar rounded shapes of an apple and a pear may elicit comparison of the two objects. The alignment of the two objects along this one salient dimension may lead children to access additional alignable features of the two objects that may not have been immediately evident. These additional alignable commonalities may be perceptual (such as that they both have stems and are both shiny) as well as conceptual (such as that they are both edible, both grow on trees, and are both served at snack time). It is important to note that even the conceptual properties that are gleaned through this process are perceptually grounded in that they are structural or relational properties abstracted from the perceptual comparison.

In the present experiments we tested two predictions that follow from this comparison-based account of categorization. First, we proposed that alignment is the specific mechanism by which comparison gives rise to the discovery of conceptual commonalities. However, this apparent alignment advantage could conceivably result from some other process; for example, comparison might trigger access to existing core beliefs about each of the objects individually as opposed to online heightening of attention to relational structure. If so, we might expect to find heightened category responding even when the two standards are from two completely different categories, simply because the standards underwent closer scrutiny. If, however, alignment is the mechanism by which children extract a category abstraction from a set of referents, then children's extensions of a given word should reflect the commonalities derived from that word's referents. For many of children's early lexical categories (such as basic-level categories) whose referents tend to share both surface and deep commonalities, this results in a benign process whereby surface similarity 
invites alignment, which in turn results in attention to the deeper commonalities that characterize taxonomic categories. However, on this alignment account, comparison may also serve to reinforce a shallow perceptual understanding if surface level commonalities are the only ones shared by the referents.

A second aspect of our proposal was that alignment is invited by common labels. If this is the case, then whether children show alignment effects should vary as a function of the manner in which the exemplars are labeled. If labeling acts as an invitation to align, then children given a common label for two exemplars should categorize according to common conceptual properties; however, if the same two exemplars are labeled by two different words, the alignment process may be inhibited.

The current experiments were designed to test these theoretical predictions to delineate how and when structure-mapping mechanisms underlie the development of taxonomic categories. This article describes two experiments, each of which explores preschool-aged children's categorization abilities. In all conditions, children were presented with a forced-choice task in which they were shown two standards and were asked to select another object of the same kind. In both experiments, the alternatives included a perceptual match that was from a contrasting taxonomic category and a category match that was perceptually distinct from the standards. In Experiment 1, we contrasted circumstances under which the standards shared both perceptual and relational features with circumstances under which perceptual commonalities were the only features shared between the two standards. In Experiment 2, we contrasted circumstances in which the two standards received a common label with those in which the standards received two conflicting labels.

\section{Experiment 1}

The structural alignment view argues that comparison yields mapping of abstract commonalities. This argument is counter to a traditional stimulus generalization account, which would predict that viewing two highly similar objects would only heighten reliance on the surface-level characteristics shared by the two objects. The evidence presented by Gentner and Namy (1999) and others demonstrates that the process of comparison facilitates mapping of commonalities at a deep, structural level of representation. It follows from this argument that in cases in which the two items share no such abstract relational commonalities, comparison will not yield similarities that would shift children's attention toward category-based responding. In fact, this manipulation may actually serve to heighten attention to perceptual commonalities.

The critical aspect of this model that we sought to test in Experiment 1 is the specificity of the alignment effect. We predicted that the product or outcome of engaging in comparison would either facilitate or inhibit attention to taxonomic category structure, depending on the commonalities available between the two exemplars. We hypothesized that when children compare two members of a taxonomic category, perceptual commonalities facilitate the abstraction of deeper relational commonalities, thus increasing the likelihood of children classifying on the basis of conceptual features. However, we hypothesized that when children compare two objects that share only perceptual features and not conceptual features, the perceptual commonalities should become even more entrenched, thus increasing the likelihood of children classifying on the basis of perceptual features, independent of category structure.

\section{Method}

Participants. Twenty-four 4-year-olds ( $M$ age $=4$ years, 5 months, range $=3$ years, 9 months -5 years, 0 months) participated. The children were from predominantly White upper-middle-class families in the greater Chicago area and attended a local preschool.

Materials. Fifty colored line drawings of real objects were used as stimuli. These drawings were organized into 10 sets of five cards each. Each stimulus set included three standards and two choice alternatives. Two of the standards were discriminably different members of a superordinate-level target category (e.g., an apple and a pear from the fruit category). The third standard was a member of a different object category that was perceptually highly similar to the other two standards (e.g., a lightbulb). Each participant saw only two of the three standards: either two objects from the target category or two unrelated objects, depending on the condition as exemplified in Figure 1.

The two choice alternatives were designed such that one card from each set, the perceptual match, was outside of the target category but perceptually resembled all three of the standards (e.g., a balloon). The other alternative, the category match, was from the target category but was perceptually distinct from the standards (e.g., a banana). Color of the pictures was controlled for in each set such that no standard matched either choice card in color. A complete list of stimuli can be found in Table 1. All stimuli were selected for their familiarity to children, and familiarity was assessed for each individual child as described in the Procedure section. Target categories were depicted at a variety of hierarchical levels.

Procedure. The children were seated across from the experimenter at a low table in a quiet room in the preschool. The children were randomly assigned to either the one-kind or the two-kind condition. In both conditions, children were introduced to Jo-Jo, a stuffed toy dog, and were told that they would learn Jo-Jo's special names for things. In both conditions, the experimenter labeled the two standard objects with a novel noun and then asked the children to help Jo-Jo find another one of the same kind.

In the one-kind condition, the experimenter laid out the two standards from the same category (e.g., apple and pear) and labeled them with a novel count noun: for example, "This is a blicket and this is also a blicket. See how these are both blickets?" After labeling the object, the experimenter asked the child to repeat the novel word. She then laid the two alternatives, the category choice (e.g., the banana) and the perceptual choice (e.g., the balloon) on the table and asked the child, "Can you tell me which one of these is a blicket?"

In the two-kind condition, the experimenter laid out two unrelated objects: an object from the target category and the out-of-kind standard (e.g., a pear and a lightbulb) and labeled them exactly as in the one-kind condition. The rest of the trial was identical to that in the one-kind condition: The experimenter asked the child to repeat the novel word and then to choose which alternative (e.g., the banana or the balloon) was a "blicket."

In both conditions, if the child did not respond or if the child selected both choice cards, the experimenter repeated the instructions. After the child selected one of the choice cards, the experimenter recorded the response and then presented the next trial.

The order of novel words and the item order were varied by selecting two random orders of each and counterbalancing them within each condition. Left-right placement of the two choice cards was randomly assigned for each trial.

After the child had completed all 10 trials, the experimenter again presented each of the stimulus sets and asked the child to tell her the "real" English names for the stimuli, to assess familiarity with the objects de- 
A

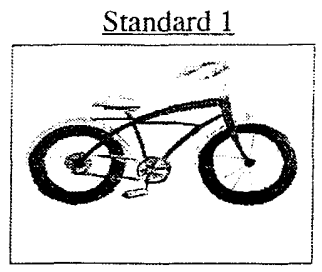

Standard 2

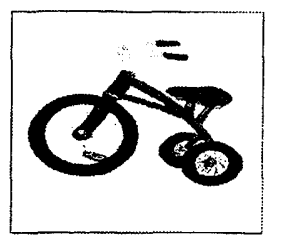

\section{Category Match}

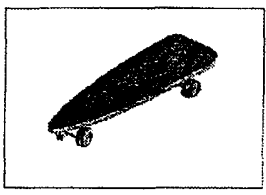

B

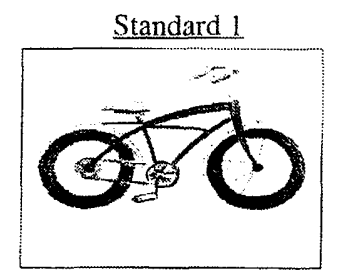

$\underline{\text { Standard } 2}$

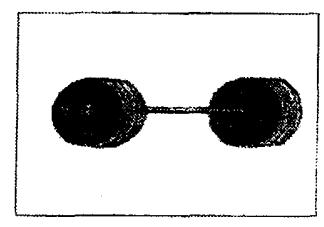

\section{Perceptual Match}

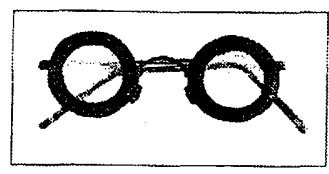

Category Match

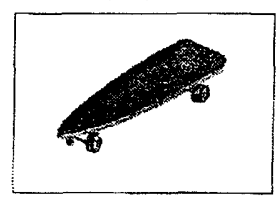

Figure 1. Sample stimulus sets. A: One-kind condition in Experiment 1 (and both conditions in Experiment 2). B: Two-kind condition in Experiment 1 .

picted. Children's names for the stimuli were evaluated by a rater blind to the experimental condition. All children tested met our inclusion criterion of providing an accurate label, synonym, or functional description for at least 35 of the 40 cards in the stimulus set viewed by the child (each child viewed only two of the three standards, resulting in a set of 40 out of 50 cards viewed by each child).

Results

We compared the proportion of trials on which children selected the category match in each condition. As predicted, the influence of comparison depended on the type of commonalities shared by the two exemplars. Children in the one-kind condition $(M=0.69$, $S D=0.24$ ) selected the category match significantly more often than those in the two-kind condition $(M=0.35, S D=0.24)$, as illustrated by $t$ tests using both participants (p) and items (i) as random factors, $t_{\mathrm{p}}(22)=3.47, p<.01, t_{\mathrm{i}}(9)=4.38, p<.01$. Children's performance on individual stimulus sets is outlined in Table 2.

Children in the one-kind condition selected the category alternatives significantly more often than predicted by chance rates $($ chance $=.50), t_{\mathrm{p}}(11)=2.73, p<.05, t_{\mathrm{i}}(9)=4.12, p<.01$. Children in the two-kind condition performed marginally below chance in their selection of the category alternatives, $t_{\mathrm{p}}(11)=2.17$, $p=.05, t_{\mathrm{i}}(9)=2.33, p<.05$. That is, when two members of a taxonomic category were given a common label, children predominantly selected the category match over a tempting perceptual match. However, when a common label was applied to two perceptually similar objects from discrepant object categories, children selected the perceptual match on $65 \%$ of trials.

We performed two additional analyses to explore these effects further. First, we examined individual children's patterns of performance in each condition. If the binomial formula is used with a total of 10 trials, an individual child must select the category match on at least 8 of the 10 trials to be reliably above chance. We found that 6 of the 12 children in the one-kind condition selected category matches at a rate that met or exceeded chance, whereas only 1 of the 12 children in the two-kind condition performed above chance. The difference between the two conditions was reliable according to the Fisher's exact test $(p=.03$ ). This suggests that the group differences reflect reliably different response patterns across individual participants within the two conditions.

Second, we examined an alternative explanation of our findings. Perhaps children selected category members more often in the one-kind than in the two-kind condition because they already possessed a lexical item for the category depicted in the one-kind condition. If so, children may simply have performed some sort of translation process, interpreting "blicket" as "fruit" for example, and then selecting another object that fell into the fruit category, without actually performing any sort of alignment. Fortunately, our stimuli allowed us to test this hypothesis, because there were some categories for which children were likely to have a lexical item, but others (such as Set 8-a bicycle, a tricycle, and a skateboard-that might be described as "nonmotorized modes of transportation" or "things you can ride around on") did not lend themselves easily to a translation process. By comparing performance of children in the one-kind condition on stimulus sets for which they were and were not likely to have pre-existing lexical items, we were able to gauge whether translation benefited the one-kind participants.

Using a conservative criterion, we identified three sets for which we suspected preschoolers were unlikely to have pre-existing labels: Sets 3 (dishware), 8 (things to ride on), and 10 (sporting equipment; see Table 1). The remaining sets were ones for which children may have had a unifying label, although they were clearly more likely to have a unifying label for some of the sets (such as 
Table 1

Stimuli Used in Experiment 1

\begin{tabular}{|c|c|c|c|c|c|}
\hline \multirow[b]{2}{*}{ Stimulus set } & \multicolumn{3}{|c|}{ Standard stimuli } & \multicolumn{2}{|c|}{ Choice alternative } \\
\hline & 1 & 2 & 3 & Perceptual & Taxonomic \\
\hline 1. Fruit & Apple & Pear & Lightbulb & Balloon & Banana \\
\hline 2. Balls & Beach ball & Baseball & Ornament & Orange & Football \\
\hline 3. Dishware & Plate & Bowl & $\mathrm{CD}$ & Cookie & Casserole dish \\
\hline 4. Musical instruments & Drum & Rounded lyre & Cake & Bucket & Flute \\
\hline 5. Vegetables & Carrot & Corn & Fish & Rocket & Turnip \\
\hline 6. Sweets & Ice cream & Lollipop & Bubble wand & Top & Candy bar \\
\hline 7. Hats & Top hat & Stovepipe hat & Candle & Soda can & Baseball cap \\
\hline 8. Things to ride on & Bicycle & Tricycle & Dumbbell & Glasses & Skateboard \\
\hline 9. Animals & Caterpillar & Snake & Belt & Rope & Turtle \\
\hline 10. Sporting equipment & Baseball bat & Golf club & Knife & Pencil & Tennis racket \\
\hline
\end{tabular}

Set 2: balls) than for others (such as Set 4: musical instruments). This analysis yielded no effect of potential labels on performance, $t(11)=0.53, n s$. Children in the one-kind condition selected the category match on an average of $0.68(S D=0.20)$ trials for those objects that they might potentially lexicalize and $0.72(S D=0.40)$ trials for those items that they were unlikely to lexicalize. Thus, it does not appear that children's performance in the one-kind condition was mediated by translation from the novel label to a known label for the target category depicted.

\section{Discussion}

The outcome of this experiment serves to specify and delineate the comparison effect. It provides support for the argument that comparison invokes an alignment process that promotes attention to commonalities. In both conditions, providing a common label for two perceptually similar objects invited alignment, but the results of this process differed by condition. In the one-kind condition, perceptual similarity was allied with conceptual similarity, and the alignment process highlighted the deeper relational commonalities depicted in the category match. In contrast, children in the two-kind condition found themselves on a dead-end street. They were only able to derive perceptual commonalities

Table 2

Proportion of Children's Taxonomic Responses for Each Set, in Each Condition in Experiment 1

\begin{tabular}{lcc}
\hline & \multicolumn{2}{c}{ Condition } \\
\cline { 2 - 3 } \multicolumn{1}{c}{ Stimulus set } & One kind & Two kind \\
\hline Fruit & .67 & .58 \\
Balls & .50 & .17 \\
Dishware & .67 & .00 \\
Musical instruments & .67 & .42 \\
Vegetables & .67 & .33 \\
Sweets & 1.00 & .50 \\
Hats & .75 & .17 \\
Things to ride on & .83 & .33 \\
Animals & .50 & .67 \\
Sporting equipment & .67 & .33 \\
\multicolumn{1}{c}{$M$} & .69 & .35 \\
\hline
\end{tabular}

because there were no deeper relational commonalities to be found.

In the next experiment, we tested another key tenet of our theory. We had postulated that common labels invite the comparison process. Our first hint that common labels invite alignment was the data reported by Gentner and Namy (1999), in which we compared the facilitative effects of comparison when we labeled the two standards with the effects of comparison when the standards were explicitly compared but not labeled. Results indicated that the effect of comparison on performance was more robust in the presence of a label than when the standards were compared but not labeled. This finding suggests that providing the same name for two standards invites children to compare them. There was, however, an overall (albeit weaker) effect of comparison in the nolabel condition as well. In the present study, we made an additional prediction that followed from the claim that labels invite alignment; namely, that giving the two standards different names should diminish any impulse to seek commonalities. Thus, whereas applying common labels should invite structural alignment and enhance the beneficial effects of comparison, applying different labels to the two standards should inhibit comparison processes, preventing the children from benefiting from the availability of two standards.

In Experiment 2, we explicitly tested this claim. We contrasted children's performance when two members of a target category were given a common label (as in the one-kind condition) with children's performance when the same two exemplars were labeled with two different words. If labeling plays an important role in inviting alignment, then alignment should be facilitated in the first case and inhibited in the second. We predicted that, as in the one-kind condition in Experiment 1, children given a common label for both exemplars would systematically select the category match. In contrast, when the two standards are given two different labels, it is possible that children could respond randomly out of confusion. However, we thought it more likely that one of two processes would occur: (a) children would focus on just one standard, ignoring the other, in which case the novel word would be extended to the perceptual alternative (as in Gentner \& Namy, 1999) or (b) more speculatively, children would engage in alignment (because of the salient perceptual commonalities between the two standards) but terminate the alignment at an early stage (because of the conflicting labels), leading them to attend to 
shallow perceptual commonalities (Goldstone, 1994a, 1994b; Love, Rouder, \& Wisniewski, 1999; A. B. Markman \& Gentner, 1993). In either case, conflicting labels should result in children selecting the perceptual alternative over the category match.

\section{Experiment 2}

In Experiment 2, we examined whether comparison is influenced by the presence of unifying versus conflicting labels. We administered a categorization task in which children were given the opportunity to compare two standards, but we manipulated how we labeled the standards. We introduced the two standards with either a single, unifying label that applied to both standards or with separate conflicting labels for each standard. If the presence of conflicting labels inhibits comparison, then children in the conflicting condition should select perceptual matches more often than those in the unifying condition. To the extent that children's comparison and abstraction processes function independent of verbal input, children should be equally likely to select the taxonomic match in both conditions.

\section{Method}

Participants. Forty 4 -year-olds ( $M$ age $=4$ years, 4 months, range $=3$ years, 9 months -5 years, 0 months) participated. The children were from predominantly White upper-middle-class families in the greater Chicago area. Their parents had responded to direct mailings or newspaper advertisements.

Materials and procedure. The stimuli and procedure were identical to those used in the one-kind condition from Experiment 1. Only the wording differed slightly. The children were randomly assigned to the unifyingword or the conflicting-word condition. In the unifying-word condition, the experimenter labeled the two standards with a single unifying novel noun: for example, "This is a blicket and this is a blicket." After labeling the object, the experimenter asked the child to repeat the novel word. She then laid the two alternatives on the table and asked the child, "Can you tell me? Which one is the same kind as these (gesturing to the standards)?"

In the conflicting-word condition, the experimenter labeled the two standards with two different contrasting novel nouns: for example, "This is a blicket, and this is a daxen." The experimenter asked the child to repeat the novel words and then laid the two alternatives on the table and asked the child, "Can you tell me? Which one is the same kind as these (gesturing to the standards)?"

\section{Results}

We compared the proportion of trials on which children selected the category match across each condition. Children in the unifyingword condition $(M=0.58, S D=0.29)$ selected the category match more frequently than did those in the contrasting-word condition $(M=0.37, S D=0.28) . T$ tests across participants and items indicated a significant effect of condition, $t_{\mathrm{p}}(38)=2.42, p<$ $.05, t_{\mathrm{i}}(9)=4.40, p<.01$. Children's performance on individual items is outlined in Table 3.

Performance in the unifying condition was only marginally above chance responding (.50) according to item analysis, $t_{\mathrm{i}}(9)=2.14, p=.06$, and did not differ from chance according to participant analysis, $t_{\mathrm{p}}(19)=1.28, p>.05$. Children in the contrasting condition selected the category match less often than predicted by chance by both item and participant analyses: $t_{\mathrm{i}}(9)=3.02, p<.05 ; t_{\mathrm{p}}(19)=2.14, p<.05$. However, support for
Table 3

Proportion of Children's Taxonomic Responses for Each Set, in Each Condition in Experiment 2

\begin{tabular}{lcc}
\hline & \multicolumn{2}{c}{ Condition } \\
\cline { 2 - 3 } \multicolumn{1}{c}{ Stimulus set } & Unifying word & Contrasting word \\
\hline Fruit & .60 & .40 \\
Balls & .50 & .35 \\
Dishware & .60 & .35 \\
Musical instruments & .40 & .45 \\
Vegetables & .65 & .20 \\
Sweets & .70 & .55 \\
Hats & .75 & .35 \\
Things to ride on & .65 & .60 \\
Animals & .40 & .20 \\
Sporting equipment & .55 & .20 \\
& & .37 \\
\hline
\end{tabular}

the prediction that the unifying condition was likely to elicit category responding was found in individual differences in children's patterns of performance in each condition. Using the binomial formula, we found that 7 of the 20 children in the unifyingword condition selected the category matches at a rate that met or exceeded chance according to the binomial (a stringent test). In contrast, only 2 of the 20 children in the contrasting-word condition performed above chance. This effect of condition on individual children's performance was marginally reliable according to Fisher's exact test $(p=.054)$.

\section{Discussion}

This study indicates that giving children a unifying label for two members of an object category appears to invite alignment and thus decreases the likelihood that the children will select a perceptual match, relative to children given conflicting labels. These findings demonstrate that whether alignment occurs depends in part on the kind of language used. When alignment was implicitly discouraged by the use of conflicting labels, children responded predominantly on the basis of perceptual, rather than taxonomic, commonalities. This finding is striking because the same task administered with the same stimuli yielded two sharply contrasting patterns. These data support the claim that labeling is one primary impetus by which children come to perceive the deep commonalities that characterize taxonomic categories. Furthermore, predominantly perceptual responding in the contrasting-word condition demonstrates that labels can act to guide children away from comparison as well as toward it. Thus, labeling can determine whether children derive commonalities across a set of items. If, as these results suggest, common labels provide children with the impetus to compare items, and if comparison yields deeper commonalities, then alignment and word-learning may work together to promote conceptual learning.

\section{General Discussion}

We began with the general hypothesis that the process of comparison is central in children's learning of category labels. These two experiments extend our initial finding that comparison facil- 
itates taxonomic categorization (Gentner \& Namy, 1999) and also provide a more specific account of the mechanisms that underlie the influence of comparison on category learning. Experiment 1 verified that the effects of comparison are specific; when the two colabeled standards shared higher order conceptual commonalities, comparison yielded heightened taxonomic performance. However, when the colabeled standards shared only surface-level commonalities, the alignment process served only to heighten children's attention to perceptual features. The results of Experiment 2 highlight the importance of labeling in encouraging children to compare and attend to taxonomic organization. When two standards that potentially shared substantial perceptual and conceptual commonalities were given a unifying label, children extended the label to the category match more often than when the same two standards were labeled with contrasting names. It is worth noting that unlike the children in Experiment 1, those in Experiment 2 who received a common label for two category members were given no explicit instruction to compare. A comparison of performance in the unifying-word condition of Experiment 2 (0.58 category responding) with performance in the one-kind condition of Experiment 1 ( 0.69 category responding), which was identical in procedure and stimuli except that it included the query, "See how these are both blickets?" suggests that adding this request to compare may have heightened the children's likelihood of arriving at the taxonomic commonalities. Although providing a common label and providing explicit instructions to compare appear to each elicit alignment effects on their own, thereby drawing children away from perceptual responding, offering both kinds of support for alignment together may result in stronger benefits of alignment.

\section{But Is It Alignment?}

Both the present experiments and our previous experiments (Gentner \& Namy, 1999) clearly demonstrate that children are more likely to select category matches that share relational and abstract commonalities with the standards when given the opportunity to compare. To what extent may we assert that these effects are attributable specifically to alignment and not simply to a similarity-based account that dictates that providing more information about what is included in the category yields more accurate categorization? There are three reasons for concluding that alignment is a more likely candidate mechanism than a simpler similarity model. First, we know from our previous experiments (Gentner \& Namy, 1999) that children's categorization performance when given two standards is more than the sum of its parts-that is, children's performance in this task when given both standards to compare is not predicted by their performance when given either standard alone. When given either standard alone, children predominantly chose the perceptual match, but presenting both together led to the opposite result: Children selected the relationally similar but perceptually dissimilar match. Second, as demonstrated in the present study, merely viewing two perceptually similar standards together is not sufficient to elicit category responding. Results for the conflicting-word condition of Experiment 2 show that the conceptual facilitation effect observed when children engage in comparison is blocked by the introduction of conflicting labels for the two standards. Third, there is some evidence in our data that explicit instructions or requests to compare (e.g., "See how these are the same kind of thing?") heighten the conceptual facilitation effect, providing further support for the claim that simply viewing two perceptually similar category members is not sufficient to elicit systematic category responding. It appears that it is specifically the comparison process that allows relational commonalities to be derived from the perceptually similar inputs. Further, the circumstances under which these additional insights are gleaned are precisely those predicted by an alignmentbased model of category learning (Gentner \& Medina, 1998). The present experiments served to delineate the proposed model of comparison as a mechanism of word and category learning.

Our findings regarding the influence of labeling on children's object categorization are consistent with previous findings that word learning facilitates acquisition of deep conceptual categories (S. A. Gelman \& Coley, 1990; S. A. Gelman \& E. M. Markman, 1986; S. A. Gelman \& Wellman, 1991; Golinkoff et al., 1992; Kemler Nelson, 1995; E. M. Markman, 1989; E. M. Markman \& Hutchinson, 1984; Waxman \& R. Gelman, 1986; Waxman \& Kosowski, 1990) and other nonobvious properties (see Waxman \& Klibanoff, 2000). Further, these findings, along with those from our previous experiments (Gentner \& Namy, 1999), can help resolve the conflicting findings regarding young children's shapebased versus taxonomy-based categorization behaviors. We suggest that structural alignment is a mechanism by which attention to shape and other salient perceptual features can actually facilitate taxonomic organization, provided that the perceptual commonalities co-occur with and provide information relating to deeper relational commonalities. Recent work on adult categorization and increasing neuroimaging evidence on the role of perceptual imagery in cognitive processing further suggests that perceptual information is infused into much conceptual processing about objects, actions, and events (e.g., Barsalou, 1999; Damasio, 1989; Goldstone \& Barsalou, 1998; Pulvermueller, 1999).

More broadly, our findings support a view of comparison as a general learning process. The role of alignment in cognitive development has been demonstrated in a broad range of other domains of cognitive development, including infant face recognition (Fagan, 1978), conservation tasks (R. Gelman, 1969), spatial relational tasks (Gentner et al., 1995; Kotovsky \& Gentner, 1996; Loewenstein \& Gentner, 2001), and word learning (Waxman, 1999; Waxman \& Klibanoff, 2000). This trend across multiple domains of development suggests that the alignment process is a general and crosscutting one.

\section{Pondering the Role of Perceptual Commonalities in Word Learning}

We have found that comparison enables children to attend to conceptual rather than perceptual commonalities in word learning. But one still may wonder why children who see a single item often extend a new word on the basis of purely perceptual commonalities. It seems inconceivable that, in the absence of comparison, children really believe that a bicycle and a pair of glasses are of the same kind, given that these objects are familiar to young children (as evidenced by their success at naming the objects depicted). We suggest that one reason for this perceptually based responding may be that, as Imai and Gentner have argued (Gentner \& Imai, 1995; Imai et al., 1994), children are accustomed to acquiring basic-level terms in which perceptual features are highly reliable predictors of underlying shared concepts. When children are asked to extend a 
novel word with incomplete knowledge about the relational properties of the word's referent, a perceptual response is a reasonable guess. Indeed, Imai and Gentner have speculated that young children may possess what might be called a naive "theory" of word learning that emphasizes perceptual features such as shape in word extension because they are such reliable predictors of categories (see also Landau, Smith, \& Jones, 1998).

Regardless of why children rely on perceptual commonalities as a basis for word learning, our studies suggest a mechanism by which this early "mistake" may serve as a pathway to deeper understanding. By mapping labels to perceptually similar entities that also happen to be conceptually similar, children are encouraged to align the items and derive the deeper conceptual commonalities that underlie the perceptual commonalities. We have documented a concrete mechanism by which children may use the perceptual level of processing as a springboard to gain insight into a conceptual level of organization.

On this account, children's tendency to categorize on the basis of shallow perceptual properties may mask or underestimate their ability to use conceptual information in a categorization task. For example, in Gentner's (1978) "Jiggy/Zimbo" study, children's word extensions did not always align with their conceptual understanding. Gentner (1978) found that children were clearly attentive to salient functional properties of a novel object (e.g., it dispenses candy) as evidenced both by their reliable ability to seek out candy from the correct object and by their exceptionally rapid acquisition of the label for the candy dispenser. However, their extensions of a novel word were on the basis of perceptual appearance rather than function.

Landau et al. (1998) have documented a similar failure of children to use functional information as a basis for word extension. In their study, 2-, 3-, and 5-year-old children were introduced to a single exemplar of a familiar category, such as "comb," and were shown its function (e.g., combing a doll's hair). Half the children were asked to extend the label (comb). The other half were asked to pick out objects that "the doll could use to fix her hair." They were then shown eight more objects, four of which shared shape but not function with the comb (e.g., a comb-shaped sponge) and four of which shared function but not shape (e.g., a toy plastic rake). The results were striking. In the function condition, children at all ages accurately accepted objects that shared function but not shape with the exemplar. However, in the label condition, children at all ages accepted objects that shared shape but not function with the exemplar.

Kemler Nelson $(1995,1999)$ has identified some circumstances under which functional information does enter into young children's new word meanings. However, her research also bears out the generality of the finding that word extensions are often perceptually based. The experimenter presented children with members of a novel category and demonstrated their function (e.g., transferring a ball to a chute). For half the children, the experimenter labeled the category prior to demonstrating the function. For the other half of the children, the order was reversed. All children were subsequently given a word-extension task. She found that, as in the previous studies, children who heard the label applied before seeing the function demonstrated were likely to make perceptual extensions of the word's meaning. However, children who learned the label after encountering functional infor- mation were more likely to take function information into account in their word extensions.

We suggest that one important factor in Kemler Nelson's study (1999) is that the function-first children had the opportunity to observe and compare a wide range of exemplars engaged in a common function, leading them to focus on the functional affordances as a basis for categorization. It is interesting to note that Kemler Nelson's (1999) materials were designed such that the perceptual properties were consistent with their functional affordances (e.g., a scoop-shaped object for lifting other objects). She speculated that these interconnections between perceptual features and functional relations are important in children's learningexactly what would be predicted on the basis of a structuremapping process that acts to promote common interconnected relational systems. As noted above, in cases like these, comparison provides a mechanism by which salient perceptual commonalities invite the discovery of linked relational commonalities.

\section{Limitations and Future Directions}

In this article we have focused on the function of alignment as highlighting common knowledge, but we have argued that alignment is an even more powerful mechanism than depicted here; we suggest that it may serve a knowledge-generating as well as a knowledge-highlighting function. If so, this comparison mechanism should also enable children to forge new abstract knowledge and induce novel inferences. In the present experiments, the categories depicted were all familiar to the children tested, at least at the basic level, so it is unlikely that children were inferring entirely new conceptual knowledge. In future work, it will be important to test the role of comparison in children's acquisition of entirely novel categories, that is, categories for which they have no prior knowledge or experience. Presenting novel objects for which we systematically manipulate children's functional, causal, or relational knowledge will allow us to assess the degree to which comparison may be used to acquire novel concepts as well as to highlight particular aspects of children's existing concepts.

Our data also suggest that children are more apt to compare and to derive benefits from comparison when they are explicitly invited to do so (e.g., "See how these are both blickets?") In future work, it will be important to explore the conditions under which children do and do not benefit from comparison to strengthen our argument that alignment processes are driving children's acquisition of categories. Preliminary pilot data suggest that the nature of the instructions as well as the presentation of the stimuli (e.g., simultaneous vs. serial presentation of the two bases) influences the extent to which children align the two bases.

\section{Conclusions}

Development is characterized by a fascinating interplay between factual observation and experience on the one hand and the child's burgeoning knowledge and belief systems on the other. The relation between perceptual similarity and category knowledge is one such interplay. Building a framework for how a child's course of learning develops from local understandings to a larger, more integrated system of knowledge is a central challenge for the field of cognitive development. We suggest that comparison processes 
play an integral role in the integration of perceptual and conceptual knowledge in development.

\section{References}

Baldwin, D. A. (1989). Priorities in children's expectations about object label reference: Form over color. Child Development, 60, 1289-1306.

Baldwin, D. A. (1992). Clarifying the role of shape in children's taxonomic assumption. Journal of Experimental Child Psychology, 54, 392-416.

Barsalou, L. W. (1999). Perceptual symbol systems. Behavioral \& Brain Sciences, 22, 577-660.

Bowerman, M. (1976). Semantic factors in the acquisition of rules for word use and sentence construction. In D. M. Morehead \& A. E. Morehead (Eds.), Normal and deficient child language (pp. 99-179). Baltimore: University Park Press.

Clark, E. (1973). What's in a word? On the child's acquisition of semantics in his first language. In T. E. Moore (Ed.), Cognitive development and the acquisition of language (pp. 65-110). New York: Academic Press.

Cohen, L. B., \& Oakes, L. M. (1993). How infants perceive a simple causal event. Developmental Psychology, 29, 421-433.

Damasio, A. R. (1989). Time-locked multiregional retroactivation: A systems-level proposal for the neural substrates of recall and recognition. Cognition, 33, 25-62.

Fagan, J. F., III (1978). Facilitation of infants' recognition memory. Child Development, 49, 1066-1075.

Falkenhainer, B., Forbus, K. D., \& Gentner, D. (1989). The structuremapping engine: Algorithm and examples. Artificial Intelligence, 41, $1-63$.

Gelman, R. (1969). Conservation acquisition: A problem of learning to attend to relevant attributes. Joumal of Experimental Child Psychology, 7, 167-187.

Gelman, S. A., \& Coley, J. D. (1990). The importance of knowing a dodo is a bird: Categories and inferences in 2-year-old children. Developmental Psychology, 26, 796-804.

Gelman, S. A., \& Markman, E. M. (1986). Categories and induction in young children. Cognition, 23, 183-209.

Gelman, S. A., \& Wellman, H. M. (1991). Insides and essences: Early understandings of the nonobvious. Cognition, 38, 213-244.

Gentner, D. (1978). What looks like a jiggy but acts like a zimbo? A study of early word meaning using artificial objects. Papers and Reports on Child Language Development, 15, 1-6.

Gentner, D. (1983). Structure-mapping: A theoretical framework for analogy. Cognitive Science, 7, 155-170.

Gentner, D. (1989). The mechanisms of analogical learning. In S. Vosniadou \& A. Ortony (Eds.), Similarity and analogical reasoning (pp. 199-241). London: Cambridge University Press.

Gentner, D., Brem, S., Ferguson, R. W., Markman, A. B., Levidow, B. B., Wolff, P., \& Forbus, K. D. (1997). Analogical reasoning and conceptual change: A case study of Johannes Kepler. The Journal of the Learning Sciences, 6, 3-40.

Gentner, D., \& Imai, M. (1995). A further examination of the shape bias in early word learning. Proceedings of the Twenty-Sixth Annual Child Language Research Forum, 167-176.

Gentner, D., \& Markman, A. B. (1995). Similarity is like analogy: Structural alignment in comparison. In C. Cacciari (Ed.), Similarity in language, thought and perception (pp. 111-147). Brussels, Belgium: BREPOLS.

Gentner, D., \& Markman, A. B. (1997). Structure mapping in analogy and similarity. American Psychologist, 52, 45-56.

Gentner, D., \& Medina, J. (1998). Similarity and the development of rules. Cognition, 65, 263-297.

Gentner, D., \& Namy, L. L. (1999). Comparison in the development of categories. Cognitive Development, 14, 487-513.

Gentner, D., \& Rattermann, M. J. (1991). Language and the career of similarity. S. A. Gelman \& J. P. Byrnes (Eds.), Perspectives on language and thought: Interrelations in development (pp. 225-277). New York: Cambridge University Press.

Gentner, D., Rattermann, M. J., Markman, A. B., \& Kotovsky, L. (1995). Two forces in the development of relational similarity. T. J. Simon \& G. S. Halford (Eds.), Developing cognitive competence: New approaches to process modeling (pp. 263-313). Hillsdale, NJ: Erlbaum.

Gentner, D., \& Wolff, P. (1997). Alignment in the processing of metaphor. Journal of Memory and Language, 37, 331-355.

Gick, M. L., \& Holyoak, K. J. (1983). Schema induction and analogical transfer. Cognitive Psychology, 15, 1-38.

Goldstone, R. L. (1994a). Influences of categorization on perceptual discrimination. Journal of Experimental Psychology: General, 123, 178200.

Goldstone, R. L. (1994b). The role of similarity in categorization: Providing a groundwork. Cognition, 52, 125-157.

Goldstone, R. L. \& Barsalou, L. W. (1998). Reuniting perception and conception. Cognition, 65, 231-262.

Golinkoff, R. M., Hirsh-Pasek, K., Bailey, L. M., \& Wenger, N. R. (1992). Young children and adults use lexical principles to learn new nouns. Developmental Psychology, 28, 99-108.

Holyoak, K. J., \& Thagard, P. (1989). Analogical mapping by constraint satisfaction. Cognitive Science, 13, 295-355.

Hummel, J. E., \& Holyoak, K. J. (1997). Distributed representations of structure: A theory of analogical access and mapping. Psychological Review, 104, 427-466.

Imai, M., Gentner, D., \& Uchida, N. (1994). Children's theories of word meaning: The role of shape similarity in early acquisition. Cognitive Development, 9, 45-75.

Kalish, C. W., \& Gelman, S. A. (1992). On wooden pillows: Multiple classifications and children's category-based inductions. Child Development, 63, 1536-1557.

Kemler Nelson, D. G. (1995). Principle-based inferences in young children's categorization: Revisiting the impact of function on the naming of artifacts. Cognitive Development, 10, 347-380.

Kemler Nelson, D. G. (1999). Attention to functional properties in toddlers' naming and problem-solving. Cognitive Development, 14, 77100.

Klibanoff, R. S., \& Waxman, S. R. (2000). Basic level object categories support the acquisition of novel adjectives: Evidence from preschoolaged children. Child Development, 71, 649-659.

Kotovsky, L., \& Gentner, D. (1996). Comparison and categorization in the development of relational similarity. Child Development, 67, 27972822.

Landau, B., Smith, L. B., \& Jones, S. (1988). The importance of shape in early lexical learning. Cognitive Development, 3, 299-321.

Landau, B., Smith, L. B., \& Jones, S. (1998). Object shape, object function, and object name. Journal of Memory \& Language, 38, 1-27.

Lassaline, M. E., \& Murphy, G. L. (1998). Alignment and category learning. Journal of Experimental Psychology: Learning, Memory, and Cognition, 24, 144-160.

Loewenstein, J., \& Gentner, D. (2001). Structural alignment promotes the development of mapping. Manuscript submitted for publication.

Loewenstein, J., Thompson, L., \& Gentner, D. (1999). Analogical encoding facilitates knowledge transfer in negotiation. Psychonomic Bulletin \& Review, 6, 586-597.

Love, B. C., Rouder, J. N., \& Wisniewski, E. J. (1999). A structural account of global and local processing. Cognitive Psychology, 38, 291316.

Markman, A. B. (1997). Constraints on analogical inference. Cognitive Science, 21, 373-418.

Markman, A. B., \& Gentner, D. (1993). Structural alignment during similarity comparisons. Cognitive Psychology, 25, 431-467. 
Markman, E. M. (1989). Categorization and naming in children: Problems of induction. Cambridge, MA: MIT Press.

Markman, E. M., \& Hutchinson, J. (1984). Children's sensitivity to constraints on word meaning: Taxonomic versus thematic relations. Cognitive Psychology, 16, 1-27.

Medin, D. L., Goldstone, R. L., \& Gentner, D. (1993). Respects for similarity. Psychological Review, 100, 254-278.

Oakes, L. M. (2001, April). The role of comparison in category formation in infancy. Paper presented at the 68th Anniversary Meeting of the Society for Research in Child Development, Minneapolis, MN.

Pulvermueller, F. (1999). Words in the brain's language. Behavioral \& Brain Sciences, 22, 253-336.

Rakison, D. H., \& Butterworth, G. E. (1998). Infants' use of object parts in early categorization. Developmental Psychology, 34, 49-62.

Ross, B. H., \& Spalding, T. L. (1991). Some influences of instance comparisons on concept formation. In D. H. Fisher, Jr., M. J. Pazzani, \& P. Langley (Eds.), Concept formation: Knowledge and experience in unsupervised learning (pp. 207-236). San Mateo, CA: Kaufmann.

Smith, L. B., Jones, S. S., \& Landau, B. (1992). Count nouns, adjectives, and perceptual properties in children's novel word interpretations. $D e$ velopmental Psychology, 28, 273-286

Tomikawa, S. A., \& Dodd, D. H. (1980). Early word meanings: Perceptually or functionally based? Child Development, 51, 1103-1109.
Ward, T. B., Vela, E., Peery, M. L., Lewis, S. N., Bauer, N. K., \& Klint, K. A. (1989). What makes a vibble a vibble? A developmental study of category generalization. Child Development, 60, 214-224.

Waxman, S. R. (1999). Specifying the scope of 13-month-olds' expectations for novel words. Cognition, 70, B35-B50.

Waxman, S. R., \& Gelman, R. (1986). Preschoolers' use of superordinate level relations in classification and language. Cognitive Development, 1 , 139-156.

Waxman, S. R., \& Klibanoff, R. S. (2000). The role of comparison in the extension of novel adjectives. Developmental Psychology, 36, 571-581.

Waxman, S. R., \& Kosowski, T. (1990). Nouns mark category relations: Toddlers' and preschoolers' word-learning biases. Child Development, 61, 1461-1473.

Wolff, P., \& Gentner, D. (2000). Evidence for role-neutral initial processing of metaphors. Journal of Experimental Psychology: Learning, Memory, and Cognition, 26, 529-541.

Received April 26, 2000

Revision received June 11, 2001

Accepted August 1, 2001 